\title{
Immune-mediated ototoxicity associated with immune checkpoint inhibitors in patients with melanoma
}

\author{
Samuel Rosner (D , , Yuri Agrawal, ${ }^{2}$ Daniel Q Sun, ${ }^{2}$ Nafi Aygun, ${ }^{3}$ \\ Megan D Schollenberger, ${ }^{4}$ Evan Lipson (D) , Jarushka Naidoo (i) ${ }^{5,7,8}$
}

To cite: Rosner S, Agrawal Y, Sun DQ, et al. Immunemediated ototoxicity associated with immune checkpoint inhibitors in patients with melanoma. Journal for ImmunoTherapy of Cancer 2020;8:e01675. doi:10.1136/ jitc-2020-001675

EL and JN contributed equally.

Accepted 22 November 2020

Check for updates

(C) Author(s) (or their employer(s)) 2020. Re-use permitted under CC BY-NC. No commercial re-use. See rights and permissions. Published by BMJ.

For numbered affiliations see end of article.

Correspondence to Dr Samuel Rosner; srosner3@jhmi.edu

\section{ABSTRACT}

Immune checkpoint inhibitors (ICls) are associated with a range of organ-specific toxicities known as immunerelated adverse events (irAEs). Immune-mediated ototoxicity from ICls is poorly described. Herein, we describe the clinical presentation, diagnostic evaluation and management of six ICl-treated patients who developed immune-mediated ototoxicity, identified by a multidisciplinary immune-related toxicity team. This is the largest case series to date and identifies bilateral highfrequency hearing loss and tinnitus as the most common reported symptoms and can be associated with abnormal speech reception thresholds and word recognition ability on audiogram in select patients. We propose multidisciplinary evaluation of patients with suspected otologic irAEs including referral to otolaryngology, audiometry evaluation \pm magnetic resonance imaging for evaluation of suspected immune-mediated ototoxicity.

\section{INTRODUCTION}

Immune checkpoint inhibitors (ICIs) that block receptors, such as cytotoxic T-lymphocyte associated protein 4 (CTLA-4) and programmed death-1 (PD-1), have led to improvements in survival for patients with metastatic disease. ${ }^{1-3}$ However, these agents are also associated with wide-ranging toxicities, termed immune-related adverse events (irAEs), which can involve various organ systems. ${ }^{4}$ Common organ-specific toxicities include pneumonitis, colitis, dermatitis, arthritis and endocrinopathies. ${ }^{5}$ Otologic irAEs have not been widely described.

Little is known about the incidence, natural history, diagnosis and management of potential ICI-mediated ototoxicity. Based on a small number of published case reports, a spectrum of clinical phenotypes of ICI-mediated ototoxicity may exist and include isolated hearing loss, tinnitus, ${ }^{6} 7$ combined hearing loss with vestibular symptoms, ${ }^{8}$ combined visual and hearing $\operatorname{loss}^{9}$ or as a complex syndrome known has Vogt-Koyanagi-Harada (VKH) leading to ocular, dermatologic and neurologic involvement. ${ }^{10-12}$
Herein, we describe the diagnostic evaluation, management and outcomes of six patients who developed immune-mediated ototoxicity, without accompanying nonotologic findings as part of a multi-organ syndrome, after receiving ICIs for metastatic melanoma.

\section{CASE DESCRIPTIONS \\ Case 1}

A 40-year-old man presented with metastatic melanoma involving the liver, spleen and bones. No intracranial metastases were seen on brain MRI. He received first-line ipilimumab (anti-CTLA-4; $3 \mathrm{mg} / \mathrm{kg}$ ) and nivolumab (antiPD-1; $1 \mathrm{mg} / \mathrm{kg}$ ). Two weeks post-ICI start, the patient developed grade 3 immune-mediated dermatitis by Common Terminology Criteria for Adverse Events (CTCAE) V.5.0, ${ }^{13}$ and grade 2 anterior uveitis requiring administration of low-dose oral prednisone (table 1). At a timepoint 3 weeks post-ICI start, while still taking prednisone for prior irAEs, he developed tinnitus and aural fullness, with bilateral moderately severe high-frequency sensorineural hearing loss (SNHL) on audiometric examination (figure 1A). Bilateral speech reception thresholds $(30 \mathrm{dbl})$ were obtained and demonstrated reduced word recognition ability (right: $88 \%$; left: 92\%). Tympanometry revealed normal middle ear pressure bilaterally. Oral prednisone was increased to a dose of $60 \mathrm{mg}$ daily, resulting in subjective hearing improvement within 2 weeks of this increase. Based on multiple irAEs and likely ICI-ototoxicity, ICI was discontinued after a single dose of each drug. The patient experienced a partial response to therapy per Response Evaluation Criteria in Solid Tumors (RECIST) V.1.1, ${ }^{14}$ ongoing at 3.5 months.

\section{Case 2}

A 61-year-old woman with melanoma of the urethra metastatic to brain was treated with 


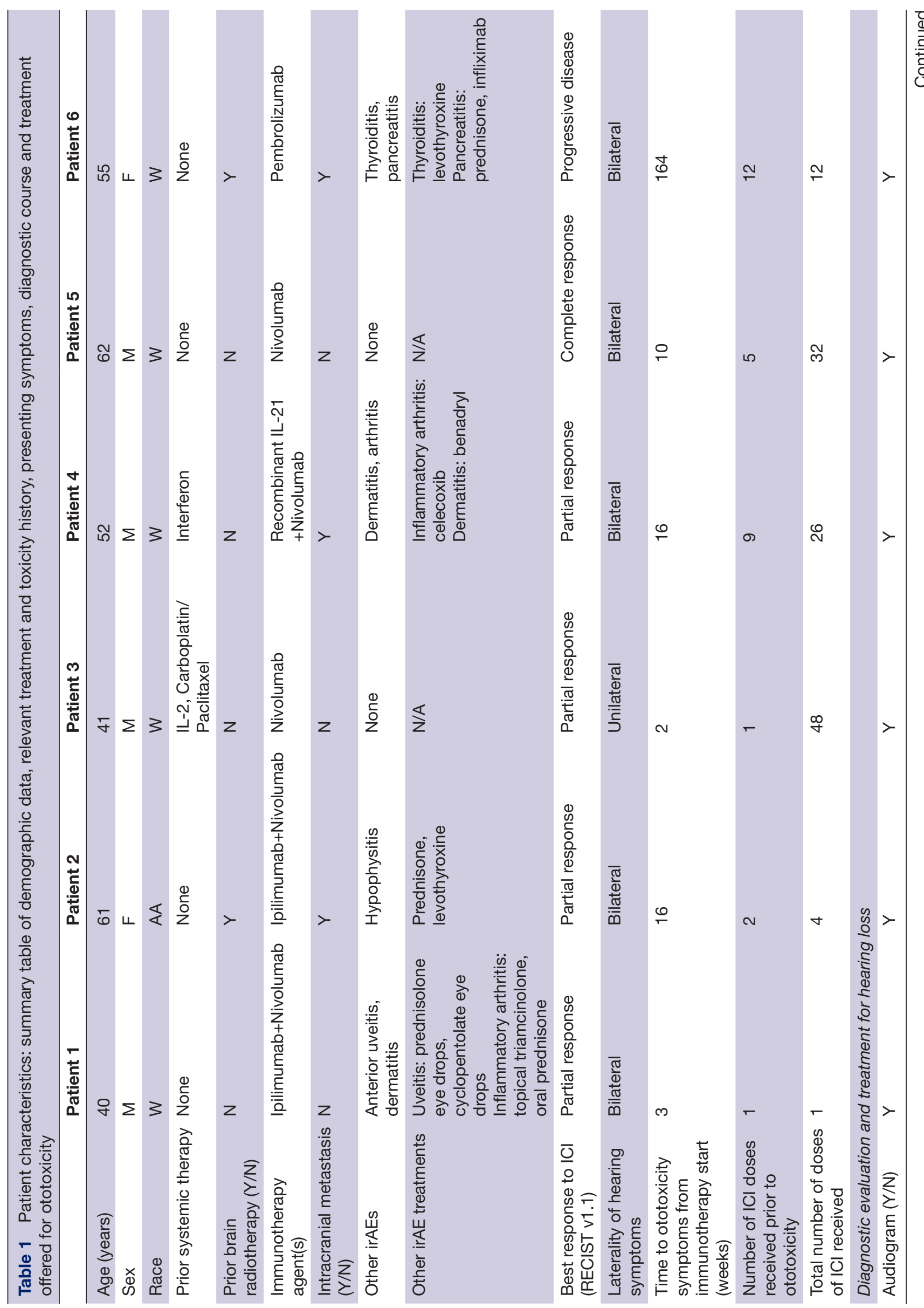




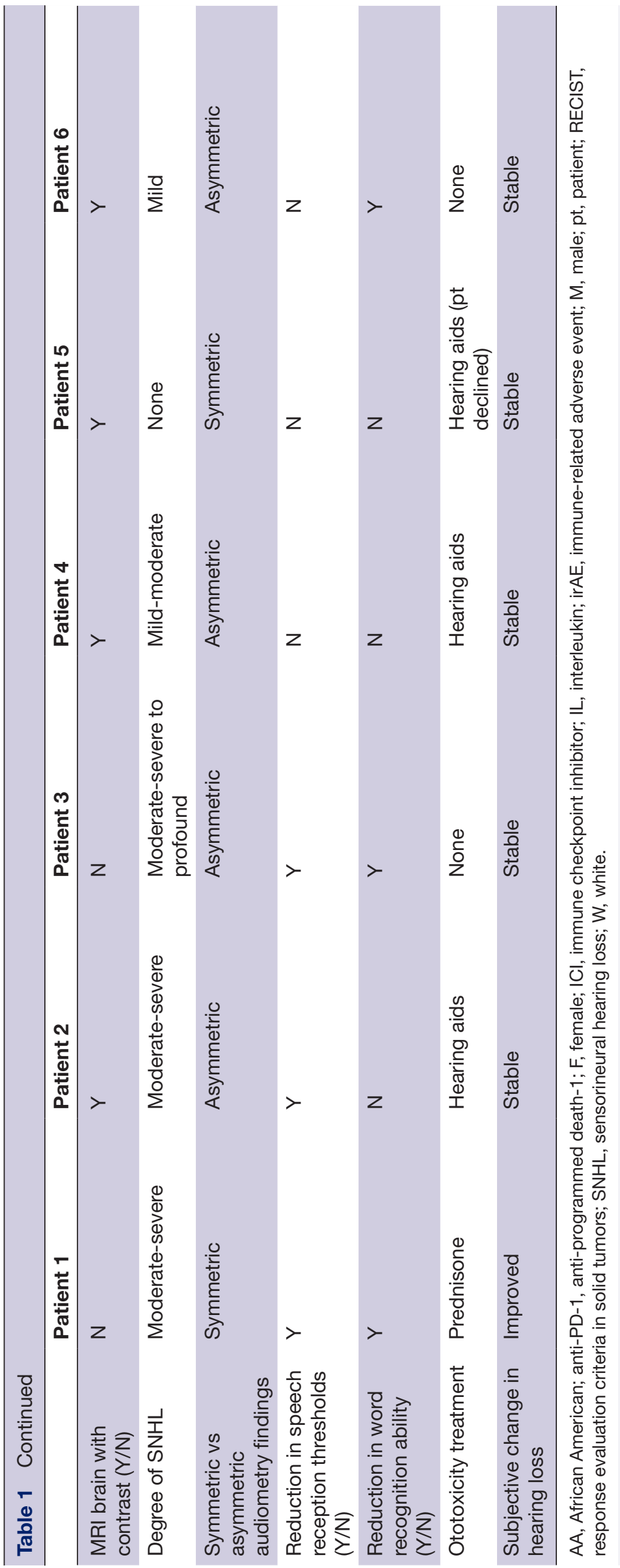




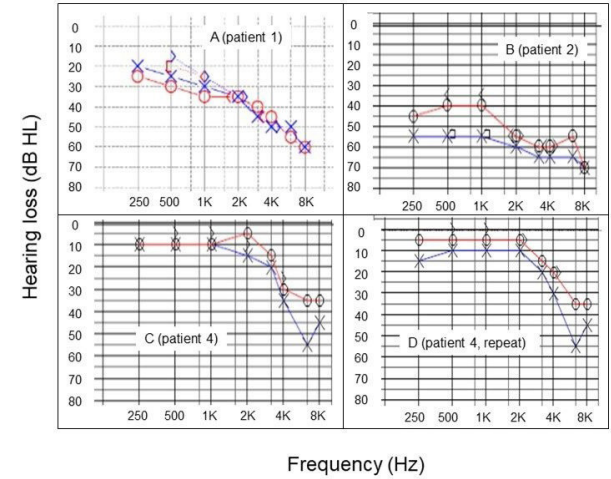

Figure 1 Post-treatment audiograms depicting hearing loss among three patients treated with ICls for metastatic melanoma. The vertical (y) axis depicts hearing loss measured in decibels in hearing level (dB HL). Zero dB HL represents the softest sound level a person with normal hearing would be expected to hear at a given frequency. The horizontal $(x)$ axis depicts sound frequency measured in hertz $(\mathrm{Hz})$. Blue line, left ear; red line, right ear. Sensorineural hearing loss is distinguished from conductive hearing loss when there is no difference between the air conduction and the bone conduction thresholds, indicating that the hearing loss is in the cochlea, as is the case for all three patients. The upper left panel (patient 1; 3 weeks into ICl) illustrates a mild sloping to moderately severe sensorineural hearing loss bilaterally. The upper right panel (patient 2; 4 months into $\mathrm{ICI})$ reveals a moderate to moderately severe sensorineural hearing loss bilaterally, L>R. The bottom panels (patient 4) represent evaluations at 4 months (left panel; normal hearing through $1500 \mathrm{~Hz}$ bilaterally, sloping to a mild sensorineural loss in the right ear and a moderate $6000 \mathrm{~Hz}$ sensorineural notch in the left ear) and 13 months (right panel; normal hearing through $4000 \mathrm{~Hz}$ sloping to a mild hearing loss in the right ear and normal hearing limits through $1500 \mathrm{~Hz}$, then sloping to a moderate sensorineural hearing loss in the left ear), ultimately revealing stable objective findings at these two separate time points. $\mathrm{ICl}$, immune checkpoint inhibitor.

first-line ipilimumab $(3 \mathrm{mg} / \mathrm{kg})$ plus nivolumab ( $1 \mathrm{mg}$ / $\mathrm{kg}$ ) and stereotactic radiosurgery to a $4 \mathrm{~mm}$ brain metastasis at the anterior putamen. At 3.5 weeks post-ICI start, after receiving two doses of combination ICI, she developed grade 2 hypophysitis treated with prednisone (table 1). Over the next 3 months, the patient reported progressive hearing loss, tinnitus and dizziness. Brain MRI demonstrated stability of the intracranial metastasis. An audiogram revealed moderate-severe bilateral SNHL (figure 1B), with reduced speech recognition threshold in the left ear, but preserved word recognition ability (right: 96\%, threshold $35 \mathrm{dbl}$; left: 100\%, threshold 50 dbl). Tympanometry results were not available. Because her hearing loss did not improve with previously administered corticosteroids, for treatment of hypophysitis, the patient's SNHL was treated with bilateral hearing aids. She experienced a partial response to ICI lasting 22 months, after which her disease progressed.

\section{Case 3}

A 41-year-old man presented with melanoma metastatic to liver and lymph nodes. No intracranial metastases were seen on brain MRI. His disease progressed after treatment with high-dose interleukin-2 (IL-2) followed by carboplatin plus paclitaxel combination chemotherapy. $\mathrm{He}$ then received nivolumab $(3 \mathrm{mg} / \mathrm{kg})$. At 2 weeks post-ICI start, the patient experienced tinnitus and leftsided hearing loss. Audiometry revealed mild-moderate SNHL through $4000 \mathrm{~Hz}$ in the right ear and mild to profound SNHL in the left ear. Speech discrimination ability was normal in the right and poor in the left ear. Over the next 5 months, the patient continued to receive ICI but noted increasing subjective hearing decline, while tinnitus was unchanged. Repeat audiometric evaluation completed at 7 months revealed mild SNHL from 2000 to $4000 \mathrm{~Hz}$ in the right ear and moderate-severe to profound SNHL, from 2000 to $8000 \mathrm{~Hz}$, in the left hear. Word discrimination was $100 \%$ in the right and $60 \%$ in the left ear. Tympanometry revealed normal middle ear pressure bilaterally. The patient's symptoms stabilized without intervention. He experienced a partial response to nivolumab lasting 31 months, after which his disease progressed.

\section{Case 4}

A 52-year-old man with metastatic melanoma received first-line recombinant interleukin-21 plus nivolumab $(3 \mathrm{mg} / \mathrm{kg})$. At 4 weeks, he developed grade 1 immunemediated dermatitis and grade 1 inflammatory arthritis (table 1). At 4 months post-ICI start, the patient reported new bilateral tinnitus. Audiometry showed mild SNHL in the right ear and moderate SNHL in the left (figure 1C). Speech reception threshold was obtained down to $10 \mathrm{~dB}$ HL bilaterally and speech recognition was $100 \%$ bilaterally. An MRI brain revealed a new $2 \mathrm{~cm}$ cerebellar mass that was treated with a single fraction of SRS, without reported changes in tinnitus symptoms. Based on the location of the intracranial lesion, it was thought an unlikely cause for his tinnitus. A repeat audiogram 7 months later demonstrated unchanged bilateral high-frequency mild to moderate SNHL (figure 1D), and the patient's hearing loss was managed with hearing aids including tinnitus maskers.

\section{Case 5}

A 62-year-old man with metastatic melanoma involving the groin and scrotum received first-line therapy with nivolumab $(3 \mathrm{mg} / \mathrm{kg})$. At 2 months post-ICI start, he developed tinnitus without subjective hearing loss. MRI brain at this time showed no evidence of intracranial metastases. ENT evaluation with audiometry revealed normal hearing sensitivity bilaterally on standard $8000 \mathrm{~Hz}$ audiogram. Speech recognition thresholds showed excellent word discrimination bilaterally. Ultra-high frequency audiometry to evaluate for more subtle abnormalities was not employed. The patient declined hearing aid placement. He continued to receive nivolumab without subjective worsening in symptoms and experienced a complete response of his metastatic melanoma, ongoing at 10 years. 


\section{Case 6}

A 55-year-old woman presented with metastatic melanoma and underwent surgical resection and SRS for brain metastases followed by first-line pembrolizumab ( $2 \mathrm{mg}$ / $\mathrm{kg})$. She experienced regression of metastatic lesions in the body but progression of brain metastases was treated with whole-brain radiotherapy (WBRT) and concomitant temozolomide, at which time pembrolizumab was held during the duration of WBRT and subsequently restarted. Her clinical course was complicated by grade 2 immunemediated thyroiditis and grade 3 pancreatitis (table 1). At approximately 3 years post-ICI start, the patient reported new bilateral ear "fullness" and decreased hearing. Brain MRI was negative for leptomeningeal disease. Audiometry showed mild left-sided SNHL (sensorineural notch at $4000 \mathrm{~Hz}$ ), $88 \%$ word recognition scoring with the right ear, $100 \%$ word recognition score with the left ear and normal tympanometry bilaterally. The patient declined symptomatic management for suspected ICI-toxicity with hearing aids. She remains without evidence of tumor progression at $\sim 4$ years post-ICI start.

\section{DISCUSSION}

Herein, we describe the largest series of patients with clinical and functional evidence of ICI-mediated ototoxicity. In each case, evidence supporting an immune-mediated phenomenon related to ICI included one or more of the following factors: (1) the temporal relationship between administration of ICI and development of symptoms, (2) symptom improvement after administration of corticosteroids and (3) absence of another causative factor (eg, a brain metastasis with apposite behavior and location).

We observed several important clinical features of ICImediated ototoxicity in this series. In terms of time to onset, patients tended to develop ICI-mediated ototoxicity early in their treatment course (within $\sim 4$ months of initiating ICI), consistent with other irAEs. ${ }^{5}$ Clinically, ICI-mediated ototoxicity manifested most commonly as bilateral tinnitus with or without high-frequency hearing loss and speech and/or word recognition deficits. Functionally, we observed that severity of ICI-mediated ototoxicity on audiogram varied widely, spanning a range of patients who exhibited objective reductions in word recognition scoring or varying degrees of SNHL to those with no abnormality on audiology. Importantly, we also identified that ICI-mediated ototoxicity could develop as a single irAE or in concert with other irAEs.

While we observed that optimal treatment in all six cases of ICI-mediated ototoxicity was unclear, in one case, symptomatic improvement was seen after administration of corticosteroids, while others were treated with hearing aids or simply observed. In cases 4,5 and 6 , steroids were not employed, as symptoms were insidious and mild without overt impairment of functional ability. Instead, symptomatic management with hearing aids was offered, with only patient in case 4 accepting this therapy with clinical improvement.
Interestingly, five of the six patients observed objective responses to ICI therapy. The association between irAEs and ICI efficacy is controversial and may depend on the site of toxicity. Retrospective studies suggest that site-specific toxicities may have a correlation with ICI efficacy. Dermatologic toxicities after PD-1 inhibitor use for metastatic melanoma have been associated with favorable response rates, ${ }^{15}$ whereas pneumonitis secondary to ICI in NSCLC corresponds with poor survival. ${ }^{16}$ For this reason, exact conclusions regarding irAE and toxicity are hard to draw and further research regarding the association of rarer irAEs, such as ototoxicity, and clinical response to ICI is needed.

Three of our six patients had presence of intracranial metastases, with two receiving some form of intracranial radiation. Evidence showing SNHL as a potential toxicity to radiotherapy have been reported. SNHL after stereotactic radiosurgery may occur in up to $1 / 3$ of cases where the inner ear is within radiation field. ${ }^{17}$ For our patient in case 2, the left anterior putamen was treated with cyberknife therapy, without inclusion of inner ear structures in the prescribed radiation field. Ototoxicity from WBRT, which was used in case 6 , is a rare toxicity with some variability based on dosing of radiation and the use of radiosensitizing agents. ${ }^{18}$ Nonetheless, preceding radiotherapy prior to onset of ototoxicity is a confounding variable. While, based on our series, ototoxicity can arise in absence of prior radiotherapy, the potential interaction between intracranial radiation and ICI warrants further study.

To date, there have been a small number of reports of patients with isolated hearing loss after receiving ICI for cancer therapy. ${ }^{6-8}$ In two reports of patients treated for melanoma, symptoms of bilateral high-frequency SNHL, corroborated by audiometry, developed within 6 weeks of initiating ICI, ${ }^{6} 7$ showing a similar acute time course to the majority of patients in our series. In a separate four-patient case series, ICIs caused a cochleovestibular toxicity in which patients had dizziness and ataxia with or without accompanying hearing loss. ${ }^{8}$ Thus, those who develop ototoxicities from ICIs may exhibit a broad spectrum of symptoms based on current reports, including ours. Last, several reports have documented ototoxicity as part of a spectrum disorder known as VKH in patients with melanoma or non-small cell lung cancer treated with ICIs, where the diagnosis largely hinged on pathognomonic fundoscopic findings. ${ }^{10-12}$ Thus, it is important to distinguish between isolated hearing changes and hearing changes as part of a collective syndrome in those with suspected ICI-mediated toxicity, and to institute appropriate management. We did not specifically evaluate ototoxicity in non-melanoma patients. However, within our institution, to our knowledge, we have not identified a non-melanoma patient treated with ICI who developed isolated ototoxicity. That being said, as noted in case series by Lemasson et al, two of their four patients with ICI-mediated ototoxicity were being treated for NSCLC, ${ }^{8}$ suggesting that this toxicity is not restricted to melanoma. 
The mechanisms underlying the development of ICImediated ototoxicity are currently unknown. Preclinical rat models of ICI-mediated ototoxicity demonstrated significant differences in auditory brainstem responses (ABR) in those treated with ICIs vs controls, as well as pathologic evidence of a reduction in the outer hair cells and flattening of the organ of Corti in those treated with ICI. ${ }^{19}$ Interestingly, ototoxicity has also been reported from adoptive cell therapy for melanoma. ${ }^{20}$ The proposed mechanism of this toxicity involves autoantigenicity against melanocyte specific antigens such as MART-1 present in the inner ear, uvea and epithelium of the skin. ${ }^{21}$

Most cases of otologic toxicity in cancer care are associated with cisplatin-based chemotherapy. ${ }^{22}$ Similar to our observed cases of ICI-mediated ototoxicity in this series, patients who develop cisplatin-induced ototoxicity present with slowly progressive SNHL. Learning from this phenomenon, these changes may be missed on standard audiograms and warrant consideration for ultrahigh frequency audiometry in consultation with an otolaryngologist, in selected cases where high-frequency hearing loss is suspected but not detected on standard clinical audiogram. ${ }^{23}$ We know that chemotherapy-induced ototoxicity is dose-dependent, due to direct damage of cochlear hair cells from reactive oxygen species, ${ }^{24}$ and is usually permanent. ${ }^{25}$ However, the relationship between ICI dose and ototoxicity as well as long-term outcomes are unknown.

This series has several limitations. As a single-institution retrospective report, we recognize that identification of cases may be subject to both selection and recall bias and may not represent the experience of other centers. A lack of baseline audiograms for each patient created challenges for distinguishing between age-related hearing loss and treatment specific changes on post-ICI audiometry. Additionally, it may have been helpful to have brain imaging at the time otologic symptom development in all cases to definitively rule out intracranial cancer progression.

In summary, we report the largest case series of patients with metastatic melanoma who developed ototoxicity in association with ICI therapy. Our report and others serve to highlight bilateral SNHL and tinnitus as the most prominent clinical features of this irAE. Based on our findings, treating oncologists should consider asking patients about hearing loss, tinnitus and/or ear fullness during ICI therapy. Among patients who develop hearing loss or tinnitus, brain MRI and subspecialist otolaryngology evaluation is warranted, including high-frequency audiometry with speech reception thresholds, word recognition ability and tympanometry to assess for middle ear disease. Both brain MRI and audiology are key in making this diagnosis, helping to rule out intracranial metastatic disease and quantify the severity of dysfunction.

In selected patients, with severe and functionally limiting symptoms, administration of corticosteroids may provide symptom improvement. Otherwise, hearing aid placement may be recommended. The decision of whether to hold or permanently discontinue ICI is difficult and requires further multidisciplinary discussion before providing exact guidance. Such a decision would involve weighing the severity of ototoxicity, clinical benefit they are receiving from ICI and the potential reversibility of their toxicity. Based on a small case series, it is hard to assess the utility and cost-effectiveness of obtaining baseline audiograms for all patients prior to initiating ICI but is certainly a topic worth studying, to help capture a more precise incidence and characterization of this toxicity. Larger, collaborative multidisciplinary studies conducted by oncologists, otolaryngologists and audiologists are needed to estimate the incidence of this phenomenon and explore optimal approaches for diagnosis and management.

\section{Author affiliations}

${ }^{1}$ Department of Oncology, Johns Hopkins Medicine Sidney Kimmel Comprehensive Cancer Center, Baltimore, Maryland, USA

${ }^{2}$ Otolaryngology, Johns Hopkins Hospital, Baltimore, Maryland, USA

${ }^{3}$ Radiology and Radiological Science, Johns Hopkins Hospital, Baltimore, Maryland, USA

${ }^{4}$ Department of Oncology, The Johns Hopkins University School of Medicine, Baltimore, Maryland, USA

${ }^{5}$ Oncology, Johns Hopkins University, Baltimore, Maryland, USA

${ }^{6}$ Oncology, Johns Hopkins Medicine Sidney Kimmel Comprehensive Cancer Center, Baltimore, Maryland, USA

${ }^{7}$ The Bloomberg Kimmel Institute for Cancer Immunotherapy, Johns Hopkins University, Baltimore, Maryland, USA

${ }^{8}$ Beaumont Hospital Dublin, The Royal College of Surgeons Dublin, Dublin, Ireland

Twitter Jarushka Naidoo @DrJNaidoo

Contributors EL: Bloomberg Kimmel Institute for Cancer Immunotherapy, the Barney Family Foundation, Moving for Melanoma of Delaware, The Laverna Hahn Charitable Trust and the National Cancer Institute P30 CA006973. JN: Bloomberg Kimmel Institute for Cancer Immunotherapy.

Funding The authors have not declared a specific grant for this research from any funding agency in the public, commercial or not-for-profit sectors.

Competing interests EL: Institutional research grant funding from Bristol-Myers Squibb, Merck, and Regeneron. Consultant for Array BioPharma, Bristol-Myers Squibb, EMD Serono, MacroGenics, Novartis, Merck, Regeneron, Sanofi Genzyme. JN: Research Grant: Bristol-Myers Squibb, Merck, AstraZeneca; Consultant: BristolMyers Squibb, AstraZeneca, Roche/Genentech; Honoraria: Bristol-Myers Squibb, Merck, AstraZeneca.

Patient consent for publication Obtained.

Provenance and peer review Not commissioned; externally peer reviewed.

Open access This is an open access article distributed in accordance with the Creative Commons Attribution Non Commercial (CC BY-NC 4.0) license, which permits others to distribute, remix, adapt, build upon this work non-commercially, and license their derivative works on different terms, provided the original work is properly cited, appropriate credit is given, any changes made indicated, and the use is non-commercial. See http://creativecommons.org/licenses/by-nc/4.0/.

\section{ORCID iDs}

Samuel Rosner http://orcid.org/0000-0003-4768-3921

Evan Lipson http://orcid.org/0000-0003-2976-0911

Jarushka Naidoo http://orcid.org/0000-0002-3470-8686

\section{REFERENCES}

1 Larkin J, Chiarion-Sileni V, Gonzalez R, et al. Five-Year survival with combined nivolumab and ipilimumab in advanced melanoma. $N$ Engl J Med 2019;381:1535-46. 
2 Hellmann MD, Paz-Ares L, Bernabe Caro R, et al. Nivolumab plus ipilimumab in advanced non-small-cell lung cancer. N Engl J Med 2019;381:2020-31.

3 Motzer RJ, Tannir NM, McDermott DF, et al. Nivolumab plus ipilimumab versus sunitinib in advanced renal-cell carcinoma. $N$ Engl J Med 2018;378:1277-90.

4 Brahmer JR, Lacchetti C, Schneider BJ, et al. Management of immune-related adverse events in patients treated with immune checkpoint inhibitor therapy: American Society of clinical oncology clinical practice guideline. J Clin Oncol 2018;36:1714-68.

5 Martins F, Sofiya L, Sykiotis GP, et al. Adverse effects of immunecheckpoint inhibitors: epidemiology, management and surveillance. Nat Rev Clin Oncol 2019;16:563-80.

6 Zibelman M, Pollak N, Olszanski AJ. Autoimmune inner ear disease in a melanoma patient treated with pembrolizumab. $J$ Immunother Cancer 2016;4:8

7 Hobelmann K, Fitzgerald D. A case of pembrolizumab induced autoimmune sensorineural hearing loss. J Otol Rhinol 2019;8:1-3.

8 Lemasson J, Cuzzubbo S, Doucet L, et al. Cochleovestibular toxicity induced by immune checkpoint inhibition: a case series. Eur $J$ Cancer 2019:117:116-8.

9 Voskens C, Cavallaro A, Erdmann M, et al. Anti-cytotoxic T-cell lymphocyte antigen-4-induced regression of spinal cord metastases in association with renal failure, atypical pneumonia, vision loss, and hearing loss. J Clin Oncol 2012;30:e356-7.

10 Crosson JN, Laird PW, Debiec M, et al. Vogt-Koyanagi-Harada-like syndrome after CTLA-4 inhibition with ipilimumab for metastatic melanoma. J Immunother 2015;38:80-4.

11 Tamura T, Akimoto E, Matsumoto C, et al. Vogt-Koyanagi-Harada Syndrome Induced by Pembrolizumab in a Patient with Non-Small Cell Lung Cancer. J Thorac Oncol 2018;13:1606-7.

12 Kurono Y, Takeda T, Kunimatsu Y, et al. Vogt-Koyanagi-Harada disease during chemoimmunotherapy for non-small cell lung cancer. Respirol Case Rep 2020;8:1-3.

13 National Cancer Institute. Common Terminology Criteria for Adverse Events (CTCAE) [Internet]. 5th ed, 2017. Available: https://ctep.
cancer.gov/protocolDevelopment/electronic_applications/docs/ CTCAE_v5_Quick_Reference_8.5x11.pdf [Accessed 21 Aug 2018].

14 Eisenhauer EA, Therasse P, Bogaerts J, et al. New response evaluation criteria in solid tumours: revised RECIST guideline (version 1.1). Eur J Cancer 2009;45:228-47.

15 Quach HT, Dewan AK, Davis EJ, et al. Association of AntiProgrammed cell death 1 cutaneous toxic effects with outcomes in patients with advanced melanoma. JAMA Oncol 2019;5:906-8.

16 Suresh K, Psoter KJ, Voong KR, et al. Impact of checkpoint inhibitor pneumonitis on survival in NSCLC patients receiving immune checkpoint immunotherapy. J Thorac Oncol 2019;14:494-502.

17 Jereczek-Fossa BA, Zarowski A, Milani F, et al. RadiotherapyInduced ear toxicity. Cancer Treat Rev 2003;29:417-30.

18 Tsao MN, Xu W, Wong RK, et al. Whole brain radiotherapy for the treatment of newly diagnosed multiple brain metastases. Cochrane Database Syst Rev 2018;1:CD003869.

19 Kuzucu İhsan, Baklacı D, Guler İsmail, et al. Investigation of the ototoxic effect of pembrolizumab using a rat model. Cureus 2019;11:e6057.

20 Seaman BJ, Guardiani EA, Brewer CC, et al. Audiovestibular dysfunction associated with adoptive cell immunotherapy for melanoma. Otolaryngol Head Neck Surg 2012;147:744-9.

21 Plonka PM, Passeron T, Brenner M, et al. What are melanocytes really doing all day long? Exp Dermatol 2009;18:799-819.

22 Nagy JL, Adelstein DJ, Newman CW, et al. Cisplatin ototoxicity: the importance of baseline audiometry. Am J Clin Oncol 1999;22:305-8.

23 Singh Chauhan R, Saxena RK, Varshey S. The role of ultrahighfrequency audiometry in the early detection of systemic druginduced hearing loss. Ear Nose Throat J 2011;90:218-22.

24 Rybak LP, Whitworth CA, Mukherjea D, et al. Mechanisms of cisplatin-induced ototoxicity and prevention. Hear Res 2007;226:157-67.

25 Frisina RD, Wheeler HE, Fossa SD, et al. Comprehensive audiometric analysis of hearing impairment and tinnitus after cisplatin-based chemotherapy in survivors of adult-onset cancer. $J$ Clin Oncol 2016;34:2712-20. 